\title{
Newborn Resuscitation in COVID-19
}

\section{Dear Editor,}

COVID-19, caused by severe acute respiratory syndrome coronavirus 2 (SARS-CoV-2), is highly transmissible, with its mode of spread via respiratory droplets, aerosol and direct contact. ${ }^{1}$

During its peak of transmission, 2 hospitals in New York City reported an incidence of $15 \%$ infection among pregnant women admitted for delivery. ${ }^{2}$ Neonatal transmission has been documented despite several precautionary measures. ${ }^{3}$ Horizontal transmission accounts for the bulk of early neonatal infection. However, significant viral load in the placenta, amniotic fluid and vaginal secretions have been reported, ${ }^{4,5}$ indicating possibility of vertical transmission in certain mother-child dyads. Although breast milk is generally considered unlikely to transmit the virus, evidence of significant viral load in breast milk exists, ${ }^{6}$ prompting caution in the face of developing COVID-19 knowledge. ${ }^{7}$

Members of the Neonatal group, Paediatric subcommittee, Singapore Resuscitation and First Aid Council convened to address precautions for Singapore's clinical practice at standby and during the resuscitation of infants born to mothers with suspected or confirmed COVID-19 infection. The recommendations are compiled based on evidence and pluralistic viewpoints from several countries and renowned bodies. ${ }^{8,9}$

Adequate personal protective equipment (PPE) is the only way to prevent neonatal responders from SARS-CoV-2 infection. ${ }^{10,11}$ Recommendations for PPE are based on 3 key factors: mode of spread of the virus, characteristics of the patients, and the role of the providers. During labour and delivery, an infected woman can disseminate the SARS-CoV-2 virus through droplets, aerosols, body fluids, and fomites. Where respiratory support is required at delivery, spread through aerosolisation is increased. Providers involved in aerosol generating procedures (AGP) such as intubation, open airway suctioning, surfactant administration, and application of nasal cannula interface at flow of $>2 \mathrm{~L} / \mathrm{min}$ are at higher risk of exposure to the SARS-CoV-2 virus. ${ }^{12}$

Hence, all personnel attending neonatal resuscitation should don N95 particulate respirator masks, goggles or face shield, full-length water-resistant gowns and gloves.
Personnel assigned with AGP should consider wearing a powered air purifying respirator (PAPR). All providers should undergo training in donning and doffing, and simulation of code situations with full PPE to familiarise with the facility, access, special precautions and communication.

Pre-assigned facility for delivery, functional equipment, trained personnel and an established workflow are requisites for safe and effective resuscitation.

Women with suspected or confirmed COVID-19 infection in labour should be cared for in a negative pressure room or isolation room, if available. ${ }^{12}$ High-risk pregnancies should be right-sited to a tertiary facility. Where dedicated space is unavailable, door to labour room should be closed at all times and compliance to infection control measures enforced. Donning of mask by the patient is recommended.

The resuscitaire should be placed at least 2 metres away from the mother if an adjacent room for neonatal resuscitation is unavailable.

In addition to antepartum and intrapartum risk factors, COVID-19 status should be communicated to the neonatal team upon admission of the mother. Discussion with the expectant mother should include: risk of infection to the newborn, measures to minimise infection risk, pros and cons of skin-to-skin at delivery, options for postpartum care, feeding options and disposition of baby.

Where high-risk delivery is expected, a designated team, limited to 3 personnel should be in attendance-a neonatal nurse and 2 medical personnel, one of whom is experienced in advanced resuscitation. Additional help may be readied for activation outside the delivery room. For low-risk delivery where the need for resuscitation is not anticipated, a single neonatal responder may remain available outside the delivery area fully donned and ready to be activated.

Before delivery, pre-brief among team members should identify the risk factors, clarify roles of the team members, and include a check of the resuscitation equipment. A personnel not directly involved in the resuscitation may be assigned to ensure appropriate donning and doffing of PPE among team members. 
To prevent wastage through contamination, minimise the equipment laid out on a resuscitaire. A recommended alternative is to pack the warming, airway and breathing or circulation equipment in separate sealed plastic bags, ready to be opened only if required. Bulb suction, high-efficiency particulate air (HEPA) filters and video laryngoscope are useful equipment to include at these deliveries.

Equipment checklist according to estimated weight or gestation, and resuscitation medication chart with predetermined volume for different weight ranges are recommended items in the resuscitation zone. These charts are useful as communication is likely to be difficult with use of N95 masks or PAPR.

An emergency resuscitation cart may be stationed outside the delivery room and extra equipment may be brought in only when needed. A transport isolette or incubator (with attached transport ventilator if baby is anticipated to require respiratory support) should be prepared and ready for use. Equipment used should be cleansed following institutional infection control guidelines.

The resuscitation algorithm and basic principles of newborn stabilisation remain unchanged. ${ }^{13}$ Key considerations are highlighted in the algorithm (Fig. 1).

There is no clear evidence of increased risk of vertical transmission through delayed cord clamping, ${ }^{14}$ neither is there definitive proof of its safety. Immediate cord clamping (ICC) reduces contact time of the newborn with maternal body fluids, potentially reducing viral transmission. However, given no clear evidence ${ }^{14}$ of risks and benefits in COVID-19, it is recommended that ICC and skin-to-skin be discussed with the expectant mother prior to delivery.

If the baby is vigorous at birth with no resuscitation needed, we advocate moving the baby to the resuscitation area under the radiant warmer for warmth and routine care. Mothers who prefer skin-to-skin with baby after delivery should be supported. However, she should wear a surgical mask and follow strict hygiene precautions. Towels used at delivery should be discarded following institutional infection control recommendations.

The initial steps of providing warmth, maintaining the open airway and stimulation are unchanged. To reduce generation of aerosols, bulb suctioning is preferred (if needed) over mechanised continuous suctioning.

In facilitating breathing, continuous positive airway pressure (CPAP) via face mask may be delivered to improve alveolar recruitment. Nasal prong bubble CPAP is discouraged as it may be aerosol generating.
In providing positive pressure ventilation, a disposable self-inflating bag or T-piece attached with HEPA filter with the lowest dead space is recommended. ${ }^{9}$ Endotracheal intubation or laryngeal mask should be considered if bag and mask ventilation is ineffective and/or during prolonged resuscitation. To ensure rapid success, it is recommended that intubation is performed by the most experienced team member. The use of a video laryngoscope allows a longer distance between the physician's and newborn's face, hence recommended if available.

Indications for chest compressions and medications remain unchanged. Where adrenaline is indicated, intravenous adrenaline $(1: 10,000$ or $0.01 \mathrm{mg} / \mathrm{ml}$ strength) is preferred over endotracheal route to prevent aerosol generation during disconnection of endotracheal tube. It is advisable that medications are prepared before the delivery when advanced resuscitation is anticipated. The runner nurse should stay outside the room to hand over any additional equipment or drugs. Communication with resuscitation team may be performed over 2-way speakers or whiteboards.

Newborns should be transported from the delivery suite in closed incubators, with accompanying staff in full PPE. A dedicated transport route and elevator should be identified and cleared for the transfer. The route should have surface decontamination following the transfer.

COVID-19 status by itself does not alter the admission and disposition guidelines following resuscitation and stabilisation of the newborn. Newborns who are unwell should be nursed in an isolation room or negative pressure room (if available) especially if respiratory support is required. For low risk and well babies, WHO recommends breastfeeding and skin-to-skin care. ${ }^{14}$ With adherence to infection control measures such as masking, hand hygiene by the mother and maintenance of 2-metre distance other than for breastfeeding, rooming in and breastfeeding may be supported.

Recommendations on resuscitation of newborns born to women with COVID-19 infection will continue to evolve with emerging evidence. This guideline allows parents to engage with healthcare workers towards decisions on resuscitation and immediate newborn care.

\footnotetext{
Acknowledgements

The authors would like to acknowledge the following contributions: Singapore Neonatal Resuscitation Course (for adaptation of the neonatal resuscitation algorithm); Yeo Kee Thai (for COVID-19 workflows at Department of Neonatology, KK Women's and Children's Hospital); Kong Juin Yee (for COVID-19 related simulation at Department of Neonatology, KK Women's and Children's Hospital); Zubair Amin (for COVID-19 workflows in resuscitation at Department of Neonatology, Khoo Teck Puat-National University Children's Medical Institute,
} 
Fig. 1. Newborn resuscitation algorithm for babies born to mothers with suspected or confirmed COVID-19 infection. Adapted and reproduced with permission from Singapore Medical Journal. ${ }^{13}$

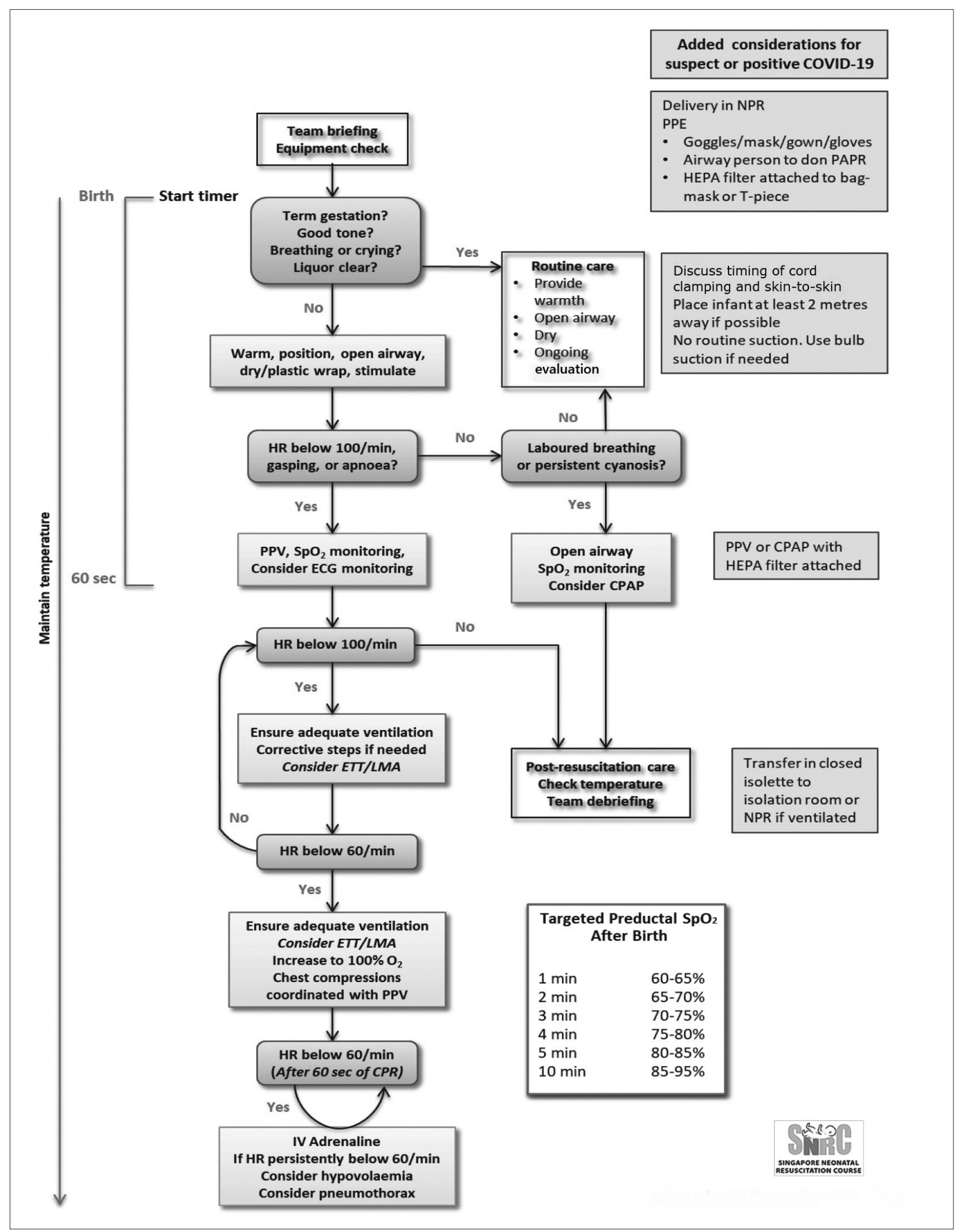

NPR: negative pressure room, PAPR: powered air purifying respirator. 
National University Hospital); Chinnadurai Amutha (for COVID-19 related simulation and workflows at Department of Neonatology, Khoo Teck PuatNational University Children's Medical Institute, National University Hospital); Khadijah Binti Abdul Kader (for COVID-19-related simulation and workflows at Department of Neonatology, Khoo Teck Puat-National University Children's Medical Institute, National University Hospital); Selina Kah Ying Ho (for COVID-19 workflows in resuscitation at Department of Neonatal and Developmental Medicine, Singapore General Hospital); Srabani Samanta Bharadwaj (for COVID-19 workflows in resuscitation and simulation at Department of Neonatal and Developmental Medicine, Singapore General Hospital); and Ereno Imelda Lustestica (for COVID-19 workflows at Department of Neonatal and Developmental Medicine, Singapore General Hospital).

\section{REFERENCES}

1. World Health Organization. Transmission of SARS-CoV-2: implications for infection prevention precautions: scientific brief, 9 July 2020. Available at: https://apps.who.int/iris/handle/10665/333114. Accessed on 30 July 2020.

2. Breslin N, Baptiste C, Gyamfi-Bannerman C, et al. Coronavirus disease 2019 infection among asymptomatic and symptomatic pregnant women: two weeks of confirmed presentations to an affiliated pair of New York City hospitals. Am J Obstet Gynecol MFM 2020;2:100118.

3. Goh XL, Low YF, Ng CH, et al. Incidence of SARS-CoV-2 vertical transmission: a meta-analysis. Arch Dis Child Fetal Neonatal Ed 2020 Jun 25;fetalneonatal-2020-319791.

4. Vivanti AJ, Vauloup-Fellous C, Prevot S, et al. Transplacental transmission of SARS-CoV-2 infection. Nat Commun 2020;11:3572.

5. Zamaniyan M, Ebadi A, Aghajanpoor S, et al. Preterm delivery, maternal death, and vertical transmission in a pregnant woman with COVID-19 infection. Prenat Diagn 2020 Apr 17:10.1002/pd.5713.

6. Gross R, Conzelmann C, Müller JA, Stenger S, et al. Detection of SARS-CoV-2 in human breastmilk. Lancet 2020;395:1757-8.

7. Lim TK. The facts, fallacies and uncertainties about coronavirus disease 2019 (COVID-19). Ann Acad Med Singap 2020;49:343-5.

8. American Academy of Pediatrics. FAQs: Management of Infants Born to Mothers with Suspected or Confirmed COVID-19, 2020. Available at: https://services.aap.org/en/pages/2019-novel-coronaviruscovid-19-infections/clinical-guidance/faqs-management-of-infantsborn-to-covid-19-mothers/. Accessed on 27 July 2020.

9. Edelson DP, Sasson C, Chan PS, et al. Interim Guidance for Basic and Advanced Life Support in Adults, Children, and Neonates With Suspected or Confirmed COVID-19: From the Emergency Cardiovascular Care Committee and Get With The Guidelines-
Resuscitation Adult and Pediatric Task Forces of the American Heart Association. Circulation 2020;141:e933-43.

10. Centers for Disease Control and Prevention. Interim Infection Prevention and Control Recommendations for Healthcare Personnel During the Coronavirus Disease 2019 (COVID-19) Pandemic, 15 July 2020. Available at: https://www.cdc.gov/ coronavirus/2019-ncov/hcp/infection-control-recommendations.html. Accessed on 30 July 2020.

11. Tan JL, Tay VS, Li H, et al. Otolaryngology Surgery in Time of COVID-19-What PPE to Use When? Ann Acad Med Singap 2020;49:387-92.

12. Tran K, Cimon K, Severn M, et al. Aerosol generating procedures and risk of transmission of acute respiratory infections to healthcare workers: a systematic review. PloS One 2012;7:e35797.

13. Yeo CL, Biswas A, Ee TT, et al. Singapore Neonatal Resuscitation Guidelines 2016. Singapore Med J 2017;58:391-403.

14. World Health Organization. Breastfeeding and COVID-19: scientific brief, 23 June 2020. Available at: https://apps.who.int/iris/ handle/10665/332639. Accessed on 30 July 2020.

Bin Huey Quek, ${ }^{* 1,2,3,4}$ MMed (Paeds), MRCP (UK), Agnihotri Biswas, ${ }^{* 3,4,5}$ MRCPCH (UK),

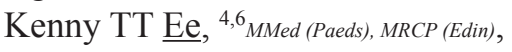
Cheo Lian $\underline{\text { Yeo, }},{ }^{2,3,4,7,8}{ }_{\text {MMed (Paeds) }}$

${ }^{1}$ Department of Neonatology, KK Women's and Children's Hospital, Singapore ${ }^{2}$ Duke-NUS School of Medicine, Singapore

${ }^{3}$ Department of Paediatrics, Yong Loo Lin School of Medicine, National University of Singapore, Singapore

${ }^{4}$ Neonatal group, Paediatric subcommittee, Singapore Resuscitation and First Aid Council, Singapore

${ }^{5}$ Department of Neonatology, Khoo Teck Puat-National University Children's Medical Institute, National University Health System, Singapore

${ }^{6}$ Kinder Clinic, Singapore

${ }^{7}$ Department of Neonatal and Developmental Medicine, Singapore General Hospital, Singapore

${ }^{8}$ Lee Kong Chian School of Medicine, Singapore

*Equal contribution as Joint First Authors

Address for Correspondence: Dr Bin Huey Quek, Department of Neonatology, KK Women's and Children's Hospital, 100 Bukit Timah Road, Singapore 229899.

Email: Quek.Bin.Huey@singhealth.com.sg 